\title{
BMJ Open Effect of aspirin on HIV disease progression among HIV-infected individuals initiating antiretroviral therapy: study protocol for a randomised controlled trial
}

\author{
Tosi Mwakyandile (D) , ${ }^{1}$ Grace Shayo, ${ }^{2}$ Sabina Mugusi, ${ }^{1}$ Bruno Sunguya (D) , ${ }^{3}$ \\ Philip Sasi, ${ }^{1}$ Candida Moshiro, ${ }^{4}$ Ferdinand Mugusi, ${ }^{2}$ Eligius Lyamuya ${ }^{5}$
}

To cite: Mwakyandile T, Shayo G, Mugusi S, et al. Effect of aspirin on HIV disease progression among HIVinfected individuals initiating antiretroviral therapy: study protocol for a randomised controlled trial. BMJ Open 2021;11:e049330. doi:10.1136/ bmjopen-2021-049330

- Prepublication history and additional supplemental material for this paper are available online. To view these files, please visit the journal online (http://dx.doi.org/10.1136/ bmjopen-2021-049330).

Received 26 January 2021 Accepted 18 October 2021

Check for updates

(c) Author(s) (or their employer(s)) 2021. Re-use permitted under CC BY-NC. No commercial re-use. See rights and permissions. Published by BMJ.

For numbered affiliations see end of article.

Correspondence to Dr Tosi Mwakyandile; tosimwakys@gmail.com

\section{ABSTRACT}

Introduction An increase in cardiovascular disease (CVD) among people living with HIV infection is linked to platelet and immune activation, a phenomenon unabolished by antiretroviral (ARV) drugs alone. In small studies, aspirin (acetylsalicylic acid [ASA]) has been shown to control immune activation, increase CD4+ count, halt HIV disease progression and reduce HIV viral load (HVL). We present a protocol for a larger ongoing randomised placebo controlled trial on the effect of an addition of ASA to ARV drugs on HIV disease progression.

Methods and analysis A single-centre phase IIA doubleblind, parallel-group randomised controlled trial intends to recruit 454 consenting ARV drug-naïve, HIV-infected adults initiating ART. Participants are randomised in blocks of 10 in a $1: 1$ ratio to receive, in addition to $A R V$ drugs, $75 \mathrm{mg}$ ASA or placebo for 6 months. The primary outcome is the proportion of participants attaining HVL of $<50$ copies/ $\mathrm{mL}$ by 8,12 and 24 weeks. Secondary outcomes include proportions of participants with HVL of $>1000$ copies/ $\mathrm{mL}$ at week 24 , attaining a $>30 \%$ rise of CD4 count from baseline value at week 12, experiencing adverse events, with normal levels of biomarkers of platelet and immune activation at weeks 12 and 24 and rates of morbidity and all-cause mortality. Intention-to-treat analysis will be done for all study outcomes.

Ethics and dissemination Ethical approval has been obtained from institutional and national ethics review committees. Findings will be submitted to peer-reviewed journals and presented in scientific conferences. Trial registration number PACTR202003522049711.

\section{INTRODUCTION}

Despite the introduction of antiretroviral therapy (ART), HIV has remained a public health concern globally, and still with high burden in Eastern and Southern Africa. ${ }^{1}$ The scaling up of the ART programmes has led to general improvement of life expectancy of people living with HIV (PLHIV) and/or AIDS..$^{23}$ This, however, has brought secondary challenges that come with chronicity of the
Strengths and limitations of this study

- This study is a double-blind, randomised controlled trial and also larger than previously reported studies looking at the effect of low-dose acetylsalicylic acid (ASA) on HIV disease.

- This study involves HIV-infected individuals as controls. These are more comparable to HIV-infected individuals exposed to ASA than HIV-uninfected individuals and/or healthy volunteers.

- Adherence evaluation is limited to pill count instead of the most objective method (measuring drug levels); however, participants receive regular reminders to improve pill count as a method of measuring adherence.

HIV disease such as increased burden of non-communicable diseases, heightened morbidity and mortality, as well as further complications from non-AIDS-defining cancers, liver, pulmonary and cardiovascular diseases (CVDs), which have been on the rise. $^{23}$

CVD is now one of the top causes of morbidity and mortality among PLHIV. ${ }^{4-8}$ This is partly because PLHIV have a higher risk of CVD than the general population, the risk being even higher in those on antiretroviral (ARV) drugs. ${ }^{9}$ Indeed, previous studies have reported an association between ARV drugs and CVD. ${ }^{10}$ Other factors that have been associated with the increased risk of CVD in PLHIV include replication of HIV, a low CD4 count, prolongation of life, high prevalence of traditional risk factors for CVD, and chronically high platelet and immune activation. ${ }^{11-13}$

T-cell activation, although less clearly, and the activation of monocytes and platelets have been reported to play a role in the 
development of CVD in PLHIV. ${ }^{14}$ For example, in PLHIV, monocytes are persistently activated, and their activation is associated with HIV pathogenesis and progression, and development of CVD. ${ }^{15-17}$ T-cell activation is associated with subclinical atherosclerosis, ${ }^{18}$ while platelet activation is reported to be one of the causes of monocyte activation. ${ }^{19}$ Activation of any of these cells may be reflected by an associated plasma and/or cellular marker such as soluble CD14 (sCD14) for monocytes, soluble P-selectin (sP-selectin) for platelets and expression of CD69, CD38 and HLA-DR for T cells. ${ }^{18-21}$

In HIV-non-infected elderly individuals and those with chronic kidney disease, sCD14 has been linked to CVD such as myocardial infarction (MI). ${ }^{22}{ }^{23}$ Likewise, in PLHIV, sCD14 is associated with CVD. ${ }^{24}$ Elevated levels of sP-selectin are associated with atherosclerosis and thrombosis and are predictive of future adverse events such as MI and stroke. ${ }^{25-27}$

Percentages of activated $\mathrm{T}$ cells ${ }^{28} 29$ and plasma levels of sCD14 and sP-selectin are higher in the PLHIV as compared with those in the general population. ${ }^{19} 30$ Furthermore, even with successful treatment with ARV drugs, percentages of activated T cells ${ }^{29}$ and plasma levels of sCD14 remain higher than that in the HIV-uninfected population. ${ }^{31-34}$ There are conflicting data on the effect of ARV drugs on plasma levels of sP-selectin. In some reports, sP-selectin levels normalise to non-HIV-infected levels with ARV drugs, ${ }^{34}$ while in other reports, the levels do not decrease ${ }^{35}$ or do not normalise to non- HIV infected levels. ${ }^{3036}$ In fact, abacavir-based ARV regimen has been reported to cause enhancement of platelet activation and platelet hyperactivity. ${ }^{37-39}$ Protease inhibitor (PI)-based ART has also been implicated in increasing platelet activation with patients on PI-based ART having up to double levels of platelet activation compared with ARV naïve counterparts. ${ }^{30}$ In view of this possible drawback of ARV drugs on immune and platelet activation, an additional drug may be necessary for effective prevention of non- infectious comorbidities including CVD in HIV infection. $^{29}$

Aspirin or acetylsalicylic acid (ASA) is a drug with antiplatelet and anti-inflammatory properties. The acute role of ASA on the increased platelet and immune activation in PLHIV has been explored with conflicting findings. In two different studies, low-dose ASA appeared to lessen platelet and/or immune activation and the associated biomarkers in PLHIV, ${ }^{19} 40$ while in another study, low-dose ASA did not affect biomarkers of immune activation. ${ }^{41}$

ASA has also been reported to have beneficial effects in highly active antiretroviral therapy (HAART)naïve patients, including halting clinical HIV disease progression $^{42}$ and significantly increasing CD4 counts. ${ }^{42} 43$ However, in one study conducted in Nigeria, an ASA containing combination resulted in a higher increase in CD4 counts at 24 weeks compared with a nonASA containing combination. This increase, however, was insignificant. ${ }^{44}$ Nevertheless, the use of ASA in PLHIV is associated with other beneficial effects like decrease in p24 antigen, decrease in tumour necrosis factor alpha, improvement in body weight (together with body mass index) and haemoglobin levels. ${ }^{44}$

ASA has also been observed to possibly have virological benefit among PLHIV. In a 12-month, three- arm, placebo-controlled pilot study conducted in Zimbabwe, $300 \mathrm{mg}$ of ASA given four times a day to ARV-naïve HIVinfected patients resulted in no increase in HIV viral load (HVL) compared with an increase in HVL observed in the placebo arm of the study. ${ }^{43}$ In another study in Zimbabwe involving ARV-naïve patients with AIDS, there was a decrease in HVL in the first 6 months of a combination of $300 \mathrm{mg}$ ASA four times a day together with chloroquine and micronutrients. ${ }^{42}$ When chloroquine was stopped but ASA and micronutrients continued for an additional 3 months, there was a further decrease in median HVL. In the two studies, however, the decline or no increase in HVL cannot be attributed to ASA alone. Furthermore, these studies involved small numbers of participants and used moderate to high doses of ASA in combination with other drugs or nutrients. ${ }^{42}$ It is therefore, necessary to conduct further studies to show the effect of ASA alone on HVL in larger studies using low-dose ASA, which is relatively safer and may provide reduction in CVD risk and/or other non-AIDS complications.

Literature shows that additional therapy to ARV drugs may compromise adherence to ARV drugs among PLHIV. ${ }^{45}$ However, when additional pill is associated with significant benefit, the addition may be justifiable. For example, addition of medication for methadone maintenance therapy and antidepressants has been reported to improve adherence to ARV drugs among HIV-infected patients in some studies. ${ }^{46} 47$ Therefore, considering the reported benefits of ASA in PLHIV, we present the protocol for an ongoing single-centre phase IIA doubleblind, parallel-group randomised controlled trial to study the effect of the addition of low-dose ASA on both HIV disease progression and adherence to ARV drugs in a relatively large sample size of HIV-infected patients initiating ART. The specific objectives of the trial are comparing HVL, CD4, biomarkers of immune and platelet activation, morbidity (number of health facility/hospital visits for medical attention/care because of illness and/or number of health facility/hospital admissions) and allcause mortality in patients on ART alone or ART plus 75 mg ASA and determining the level of adherence to ARV drugs over a 6-month period in the ART plus ASA arm and the placebo arm.

We hypothesise that addition of low-dose ASA to ART in the newly recruited HIV-infected patients will be associated with earlier and sustained virological suppression and better immunological and clinical responses. Moreover, addition of low-dose ASA to ART will not compromise adherence to ARV drugs.

Previous studies that looked at the effect of ASA on HIV infection parameters such as HVL and CD4+ count in PLHIV involved the use of very high doses of ASA (up to $1800 \mathrm{mg} /$ day).$^{42-44}$ However, such high doses carry high 
risk of adverse effects such as gastrointestinal bleeding and, rarely, cerebral bleeding, which are less likely with low doses. The use of this drug at such high dose as part of routine HIV care will be potentially hazardous. On the other hand, studies that have looked at the effect of ASA on biomarkers of platelet and/or immune activation have demonstrated effect with low-dose ASA, which may be considered safe in routine care. ${ }^{19}{ }^{40}$ Additionally, lowdose ASA is known to have an antiplatelet effect and thus appropriate for suppressing platelet activation. There is increased platelet activity leading to increased immune activation in PLHIV, which is associated with the increased CVD risk. This increased platelet activity is not completely abolished by ARV drugs, ${ }^{30} 36$ and indeed, there are reports that ARV drugs may enhance platelet activation. ${ }^{30}$ 37-39 Therefore, low-dose ASA added onto ARV drugs may be the more appropriate intervention than high-dose ASA for reducing CVD risk in PLHIV.

The use of placebo as a comparator in this trial is appropriate and does not present any ethical concern because the use of low-dose ASA is not standard of care and participants in the placebo group continue receiving ARV drugs.

\section{METHODS AND ANALYSIS \\ Study design}

This trial is a single-centre phase IIA double-blind, parallel-group randomised placebo-controlled study aiming at determining the effect of low-dose ASA on virological, immunological and clinical parameters among HIV-infected individuals initiating ART.

\section{Study setting}

The study is implemented at two Management and Development for Health-supported HIV care and treatment clinics (CTCs) in Temeke district, Dar es Salaam region,
Tanzania. These sites are large volume clinics operating 6 days a week where three to five newly diagnosed HIVinfected patients are recruited daily at each site.

\section{Study population}

The study participants for this study are newly recruited HIV-infected patients initiating ART. At the time of recruitment, all participants are naïve to ARV and planned to initiate on ART. All participants should show willingness to stay in Dar es Salaam and attend the respective CTCs for at least six consecutive months. This mandate is to minimise loss to follow-up cases. Those who are asthmatic, pregnant, predisposed to bleeding, on antithrombotic therapy and therapy with prohibited drugs (see online supplemental file 1), with active or history of peptic ulcer disease, previous intolerance or allergy to ASA or any ASA products and/or severe renal disease (estimated glomerular filtration rate $<30 \mathrm{mil} / \mathrm{min} / 1.73 \mathrm{~m}^{2}$ ) are excluded (see table 1).

\section{Selection of participants}

Prospect participants are informed of the study and invited to participate by a study nurse. Those who agree to participate are prescreened for potential eligibility. Each potentially eligible participant is asked to provide a written informed consent (see online supplemental file 2) by study doctor before being screened for eligibility and those who meet all the inclusion criteria and none of the exclusion criteria are enrolled into the trial.

\section{Randomisation and blinding}

Computer-generated randomisation table for each site has been performed separately so that each site contributes equally to the minimum sample size. Eligible participants are block-randomised to either study drug, in addition to ART, in a ratio of 1:1. Each block comprises 10 participants. A randomisation schedule, prepared by

Table 1 Inclusion and exclusion criteria

\begin{tabular}{|c|c|}
\hline Inclusion criteria & Exclusion criteria \\
\hline $\begin{array}{l}\text { Consenting newly recruited male or female HIV-infected } \\
\text { patients }\end{array}$ & Previous intolerance or allergy to ASA or any ASA products \\
\hline ARV drug-naïve initiating on ARV drugs & Asthmatics \\
\hline Age 18 years or older & $\begin{array}{l}\text { Predisposition to bleeding (increased chance of bleeding due } \\
\text { to being on antiplatelets and/or anticoagulants and/or having } \\
\text { history of or active diagnosis of bleeding disorder; see online } \\
\text { supplemental file 3) }\end{array}$ \\
\hline $\begin{array}{l}\text { Willingness to stay in Dar es Salaam for at least } 6 \\
\text { consecutive months }\end{array}$ & Antithrombotic therapy \\
\hline \multirow[t]{4}{*}{$\begin{array}{l}\text { Willingness to attend HIV clinics at Temeke or Mbagala Rangi } \\
\text { Tatu hospitals for at least six consecutive months }\end{array}$} & Therapy with prohibited drugs (see online supplemental file 1) \\
\hline & Active or history of peptic ulcer disease \\
\hline & Pregnancy \\
\hline & Severe renal disease (eGFR <30 mil/min/1.73 m2) \\
\hline
\end{tabular}


an independent statistician in Boston, Massachusetts, USA, was sent electronically to an independent pharmaceutical technician stationed at the Muhimbili University of Health and Allied Sciences (MUHAS) for labelling of the study drug boxes. The randomisation schedule contained study numbers placed against their respective study letters representing the study sites and treatment groups. The pharmaceutical technician is the only person who knows the drug company codes for identification of the placebo and ASA drugs but does not have direct contact with the participants. The drug boxes are stored at MUHAS and doses for the participants are sent to the respective sites monthly where blinded clinic pharmacists dispense them. All members of the study team and the study participants are blinded to treatment allocation. Only the independent statistician and the independent pharmaceutical technician are not blinded. The randomisation code remains with the statistician until the time of report writing. Following completion of the study, the randomisation code will only be available once all collected data will have been entered into the database for every participant and the database has been finalised. During the study duration, breaking the code will only occur on recommendation by the Data and Safety Monitoring Board (DSMB). In such circumstances, the participant's study number will be sent to the independent statistician to provide the corresponding treatment allocation to the DSMB.

\section{Intervention}

Aspirin arm

In addition to their ARV regimen, participants selfadminister blister packaged enteric coated tablet of $75 \mathrm{mg}$ ASA (Cardisprin 75, Cosmos, Nairobi, Kenya) for 24 weeks at a dose of one tablet per day at evening times swallowed wholly with a glass of clean drinking water, preferably after a meal. All adults initiating ART are prescribed the default combination of tenofovir (TDF) +lamivudine (3TC)+dolutegravir (DTG). Those with contraindications are alternatively prescribed abacavir+3TC+DTG or TDF +3TC+efavirenz and in special situations zidovudine+3TC+DTG. ${ }^{48}$

\section{Placebo arm}

In addition to their ARV regimen, participants selfadminister blister packaged placebo (Cardisprin 75, Cosmos, Nairobi, Kenya) for 24 weeks at a dose of one tablet per day at evening times swallowed wholly with a glass of clean drinking water, preferably after a meal. The placebo has colour, shape and size similar to ASA.

There will be no any allowable dose modifications. However, the investigator may withdraw a patient from the study treatment procedures if the patient will be in violation of the protocol, experience a serious adverse event (SAE) that is related to the study drug, develop (during the course of the study) symptoms or conditions listed in the exclusion criteria and/or require a medication that is prohibited by the protocol. The investigator will also withdraw all participants from the study treatment if the study is terminated. Patients will be free to withdraw from the study at any time on their request. All the patients who will be withdrawn from the study will be followed up to completion of 6 months of follow-up for collection of safety data.

\section{Evaluation}

During screening for eligibility, demographic information, medical history including history of CVD and risk factors, previous and current medication, HIV diagnosis and treatment and contraindication to ASA are taken. In addition, patients go through vital signs and anthropometric measurements and a thorough physical examination. For female patients, urine for pregnancy test is done at baseline and subsequent visits to screen for pregnancy (Laborex, Zhejiang Orient Gene Biotech Co., China). After qualifying for enrolment and randomisation, participants provide blood samples for full blood picture (FBP), liver function tests (LFTs), renal function tests (RFTs), lipid profile, CD4 count, HVL, plasma levels of sCD14, sP-selectin and for markers of T-cell activation and exhaustion (CD69, CD38, HLA-DR and PD-1, respectively).

Each enrolled participant will have a total of 6 monthly visits. Assessment and tests that are done in each follow-up visit are summarised in the table 2.

All adverse events (AEs) will be recorded on a specially designed form and will be classified (mild, moderate and severe). The relationship with the study medication will be judged and the action taken will be recorded. AEs will be followed up until resolution or end of study. All SAEs, whether or not deemed drug-related, or expected, will be reported to the MUHAS and the national ethics committees within 24-72 hours of occurrence using the SAE notification form. Clinical trial participation insurance has been purchased should there be an event of death of the participant and/ or total permanent disability resulting from the study drugs but not from any other cause which has nothing to do with the study.

\section{Laboratory tests}

Different volumes of blood are drawn from each patient, depending on the visit (table 3). All samples are transported in a cool box at room temperature to the MUHAS immunology laboratory (IL) and MUHAS Clinical Research Laboratory (MCRL) for processing, storage and/or analysis. Time allowable from sample collection to processing of FBP and CD4 count is 6 and 24 hours, respectively. At the MCRL, $50 \mu \mathrm{L}$ drawn from $4 \mathrm{~mL}$ blood sample in K2 EDTA vacutainer tube is used for analysis of FBP using Sysmex analyser (Sysmex Corporation, Japan), and the rest is transported to the Infectious Disease Centre laboratory within Dar es Salaam for CD4 count (FACSPresto; BD Biosciences, San Jose, California, USA). Lipid profile, RFT and LFT are tested using COBAS Integra 400 Plus (Roche Instruments Centre AG, Switzerland) from serum obtained from centrifugation (at 2500 
Table 2 Schedule of assessments at each visit

\begin{tabular}{|c|c|c|c|c|c|c|c|}
\hline Month & Visit 0 & Visit 1 & Visit 2 & Visit 3 & Visit 4 & Visit 5 & Visit 6 \\
\hline Informed consent & $\sqrt{ }$ & & & & & & \\
\hline Screening & $\sqrt{ }$ & & & & & & \\
\hline Medical history & $\sqrt{ }$ & & & & & & \\
\hline Clinical examination & $\sqrt{ }$ & $\sqrt{ }$ & $\sqrt{ }$ & $\sqrt{ }$ & $\sqrt{ }$ & $\sqrt{ }$ & $\sqrt{ }$ \\
\hline Weight measurement & $\sqrt{ }$ & $\sqrt{ }$ & $\sqrt{ }$ & $\sqrt{ }$ & $\sqrt{ }$ & $\sqrt{ }$ & $\sqrt{ }$ \\
\hline Blood pressure measurement & $\sqrt{ }$ & $\sqrt{ }$ & $\sqrt{ }$ & $\sqrt{ }$ & $\sqrt{ }$ & $\sqrt{ }$ & $\sqrt{ }$ \\
\hline Urine pregnancy test & $\sqrt{ }$ & $\sqrt{ }$ & $\sqrt{ }$ & $\sqrt{ }$ & $\sqrt{ }$ & $\sqrt{ }$ & \\
\hline Confirm eligibility & & $\sqrt{ }$ & $\sqrt{ }$ & $\sqrt{ }$ & $\sqrt{ }$ & $\sqrt{ }$ & \\
\hline Randomisation & $\sqrt{ }$ & & & & & & \\
\hline FBP, LFT, RFT, lipid profile & $\sqrt{ }$ & & & & & & $\sqrt{ }$ \\
\hline sCD14, sP-selectin, CD69, CD38, HLA-DR, PD-1 & $\sqrt{ }$ & & & $\sqrt{ }$ & & & $\sqrt{ }$ \\
\hline Study drug dispensing & $\sqrt{ }$ & $\sqrt{ }$ & $\sqrt{ }$ & $\sqrt{ }$ & $\sqrt{ }$ & $\sqrt{ }$ & \\
\hline Adherence to ART check & & $\sqrt{ }$ & $\sqrt{ }$ & $\sqrt{ }$ & $\sqrt{ }$ & $\sqrt{ }$ & $\sqrt{ }$ \\
\hline Compliance to ASA check & & $\sqrt{ }$ & $\sqrt{ }$ & $\sqrt{ }$ & $\sqrt{ }$ & $\sqrt{ }$ & $\sqrt{ }$ \\
\hline Morbidity check & & $\sqrt{ }$ & $\sqrt{ }$ & $\sqrt{ }$ & $\sqrt{ }$ & $\sqrt{ }$ & $\sqrt{ }$ \\
\hline Adverse event check & & $\sqrt{ }$ & $\sqrt{ }$ & $\sqrt{ }$ & $\sqrt{ }$ & $\sqrt{ }$ & $\sqrt{ }$ \\
\hline Mortality check & & $\sqrt{ }$ & $\sqrt{ }$ & $\sqrt{ }$ & $\sqrt{ }$ & $\sqrt{ }$ & $\sqrt{ }$ \\
\hline Concomitant medication check & & $\sqrt{ }$ & $\sqrt{ }$ & $\sqrt{ }$ & $\sqrt{ }$ & $\sqrt{ }$ & $\sqrt{ }$ \\
\hline
\end{tabular}

ART, antiretroviral therapy; ASA, acetyl salicylic acid; FBP, full blood picture; LFT, liver function test; RFT, renal function test; sCD14, soluble CD14; sP-selectin, soluble P-selectin.

rpm for $15 \mathrm{~min}$ ) of $4 \mathrm{~mL}$ sample (in red topped vacutainer tube) within 6 hours since collection. The obtained serum is stored at $-80^{\circ} \mathrm{C}$ awaiting analysis. All the frozen samples will slowly be brought to room temperature at the time of analysis.

Table 3 Blood sampling schedule at each visit

\begin{tabular}{|c|c|c|c|}
\hline Visit & Test & Vacutainer tube & Volume (mL) \\
\hline \multirow[t]{3}{*}{ Visit 0} & FBP, CD4 count & K2 EDTA & 4 \\
\hline & $\begin{array}{l}\text { HVL, sCD14, sP-selectin, PBMCs separation for markers of T-cell activation } \\
\text { and exhaustion }\end{array}$ & K2 EDTA & 12 \\
\hline & Lipid profile, RFT, LFT & Red topped & 4 \\
\hline Visit 2 & HVL & K2 EDTA & 8 \\
\hline \multirow[t]{2}{*}{ Visit 6} & FBP, CD4 count & K2 EDTA & 4 \\
\hline & $\begin{array}{l}\text { HVL, sCD14, sP-selectin, PBMCs separation for markers of T-cell activation } \\
\text { and exhaustion }\end{array}$ & K2 EDTA & 12 \\
\hline
\end{tabular}

FBP, full blood picture; HVL, HIV viral load; LFT, liver function test; PBMC, peripheral blood mononuclear cell; RFT, renal function test; sCD14, soluble CD14; sP-selectin, soluble P-selectin. 
At the IL, $12 \mathrm{~mL}$ sample in K2 EDTA vacutainer tube for plasma and peripheral blood mononuclear cells (PBMCs) is centrifuged at 1500 times gravity $(\times g)$ for $10 \mathrm{~min}$ at minimum acceleration and deceleration to separate plasma within 2 hours from the time of sample collection. Separated plasma is aliquoted in 1.5 and 4.5 $\mathrm{mL}$ portions and immediately stored at $-80^{\circ} \mathrm{C}$. The 1.5 $\mathrm{mL}$ aliquot will later be used for HVL testing, while the $4.5 \mathrm{~mL}$ aliquot will be shipped to Kumamoto University in Japan for sCD14 and sP-selectin. sCD14 and sP-selectin analysis will be done using customised BDTM Cytometric Bead Array (BD Biosciences). Individual plex assay for human sP-selectin and sCD14 will be used to configure a multiplex system for quantification of the targeted biomarkers simultaneously.

PBMCs are separated from cellular sediment of whole blood obtained from plasma separation procedure and then stored at $-80^{\circ} \mathrm{C}$. These are also to be transported to Kumamoto University in Japan for T-cell activation and exhaustion analysis. PBMCs will be thawed and stained with live and dead fixable yellow stain (Invitrogen, Carlsbad, California, USA). Aliquots of the stained cells will then be further stained with CD3 PerCP (BioLegend, San Diego, California, USA), CD4 AF700, CD8 APC-H7, CD69 APC, HLA-DR PE, CD38 APC and PD-1 BV421 (BD Biosciences), and with CD14 Pacific Blue, CD16 PE and CD69 PE-Cy7 (BD Biosciences) and acquired on an LSRII flow cytometer (BD Biosciences) using FacsDiva software. Percentages of activated (CD69+ CD38+HLA-DR+) and exhausted (PD1+) CD4 and CD8 T cells and monocyte subsets (CD14/CD16/CD69) will be determined by gating based on isotype controls using FacsDiva software (BD Biosciences).

\section{Primary and secondary outcomes}

The primary outcome is the proportion of participants attaining HVL of $<50$ copies/mL at the end of 8,12 and 24 weeks. In addition, the difference in the proportion and the $95 \%$ CI of the difference will be calculated. Secondary outcomes include proportion of participants with HVL of $>1000$ copies $/ \mathrm{mL}$ at the end of 24 weeks, proportion of participants attaining a $>30 \%$ rise of $\mathrm{CD} 4$ count from baseline value at the end of 12 weeks, ${ }^{49}$ and prevalence of morbidity and all-cause mortality. Other secondary outcomes are proportion of participants with normal levels of the assessed biomarkers of platelet and immune activation at the end of 12 and 24 weeks, proportion of participants experiencing AEs, mean percentage adherence to ARV drugs and mean percentage compliance to the study drugs.

\section{Definitions and measurements of outcomes}

Morbidity: The number of health facility/hospital visits for medical attention/care because of illness and/ or number of health facility/hospital admissions. At each monthly visit, patients are being asked if they visited any healthcare facility to seek medical care/ treatment and/ or if they were admitted for medical reasons since last visit. The reason for visit or admission will be traced from the patient or medical and/or clinical records, respectively.

All-cause mortality: Confirmed death of any cause with certification of death by the medical practitioner or a verbal or telephone confirmation of death from a relative or friend.

Adherence to ARV drugs: Greater than $90 \%$ adherence by pill count. ${ }^{50}$

Compliance to study drugs: Adherence by pill count to study drugs for at least 20 days in a 28-day (4-week) period. $^{51}$

Patients' remaining monthly tablets of study drugs and ARV are counted to ascertain compliance and adherence, respectively, at each monthly follow-up visit. Overall compliance and/or adherence will be the average of the monthly compliances and/or adherences, respectively. For patients who will not last in the study for all the 6 months, their compliance and/or adherence will be calculated by taking the average of the monthly compliance and/or adherences, respectively, for the months they have been in the study.

\section{Data collection and management}

Data will be collected through interviewing the patients, from their medical files/treatment charts and source data documents and will be recorded on the case report form (CRF). All data collection procedures are guided by trial standard operating procedures and are done by a study team trained on the protocol and the trial operating procedures. The CRFs will be checked for completeness and accuracy against the source data. Original CRFs will be used when entering information into the computer database. The data will be double entered into a computer by two different data entry clerks and the entered data will then be verified and cleaned before being subjected to analysis using the SPSS statistical programme version 23. The database will be checked against the CRFs for accuracy. Data analysis will begin after ensuring that all the data have been accurately entered. Data will be kept in a password-secured computer to ensure confidentiality. All the blood samples will be stored at respective analysing laboratories, whereas, documents will be stored by the investigator during the study period until time for destruction after the data have been analysed and published.

\section{Patient and public involvement}

No patients were involved.

\section{Statistical considerations}

Sample size calculation

This is based on the proportion of patients on ART alone with HVL of $<50$ copies $/ \mathrm{mL}$ at 8 weeks, which is $75 \% .^{52}$ Assuming that the addition of ASA to ART will give a $15 \%$ increase in this proportion, then at a power of $90 \%, 10 \%$ loss to follow-up and $95 \%$ CI, the sample size per each arm is 227 participants. 


\section{Statistical analysis}

Intention-to-treat analysis will be done. Baseline characteristics will be summarised by treatment arms. $\chi^{2}$ or Fisher's exact test will be used for comparison of proportions. Student's t-test or Wilcoxon rank-sum test will be used for comparison of means or medians between the two arms. Descriptive statistics will also be used where appropriate. A two-tailed $p$ value of $<0.05$ will be considered significant.

The primary efficacy trial endpoint for this trial is the proportion of participants attaining HVL of $<50$ copies/ $\mathrm{mL}$ at the end of 8,12 and 24 weeks. The primary safety endpoint is gastrointestinal bleeding. $\chi^{2}$ or Fisher's exact test will be used to test the differences in the proportions of participants with HVL of $<50$ copies/mL between the treatment arms, and also to test the differences in proportions of participants experiencing AEs such as gastrointestinal bleeding.

We will use linear mixed-effects models with a random intercept and robust SEs to assess mean differences between baseline and postrandomisation levels of platelet and immune activation biomarkers by randomisation group. Linear mixed-effects models will account for within-subject correlation, and accommodate varying number and timing of measurements per participant over time.

A generalised linear mixed model with random intercept will be used to assess differences in the proportions of participants attaining a $>30 \%$ rise of $\mathrm{CD} 4$ and incidence of morbidity. Log-rank test will be used to test the differences in the incidence rates of death between the treatment arms.

\section{ETHICS AND DISSEMINATION}

The protocol was approved by the institutional ethics committee of MUHAS (reference number DA.282/298/01/C) and the National Health Research Ethics Committee at the Tanzania National Institute of Medical Research (NIMR) (NIMR/HQ/R.8a/Vol. IX/3001). Approval of the protocol and the study drugs was done by the Tanzania Medicine and Medical Devices Authority (reference number TFDA0019/CTR/0003/03 and authorisation number TZ19CT0008). Prior to enrolment in the study, a written informed consent is obtained from each patient.

This study is being conducted in compliance with the current version of the protocol. Any change to the protocol document or informed consent form that affects the scientific intent, study design and patient safety, or may affect a participant's willingness to continue participation in the study will be considered an amendment and therefore will be written and filed as an amendment to this protocol and/or informed consent form. All such amendments will be submitted to the ethics committees for approval prior to becoming effective.

The trial has a DSMB comprising a clinical pharmacologist, a physician and a biostatistician. This is an independent committee which independently monitors the study at intervals to review progress reports, safety data, trial performance and progress, and makes pertinent recommendations to research ethics committees, regulatory authority, directorate of research and publications at MUHAS, and the investigator.

Findings of this study will be sent for publication in peer-reviewed journals and also presented in international, regional and local conferences.

\section{DISCUSSION}

To the best of our knowledge, there are no studies done in the highly HIV-burdened sub-Saharan Africa that have looked at the effect of ASA on HIV infection parameters and biomarkers of platelet and immune activation concurrently.

The studies that looked at the effect of ASA in PLHIV on HIV infection parameters such as HVL and CD4+ count involved the use of very high doses of ASA (1.2-1.8 g/day in divided doses). It is reported, however, that there are risks for adverse effects such as gastrointestinal bleeding and rarely cerebral bleeding, which are more likely associated with high doses than with low doses. ${ }^{53}$ Again, these studies involved smaller numbers of participants, and ASA was given in combination with other drugs such as chloroquine and/or nutrients such as selenium and multivitamins. ${ }^{42-44}$ Our study is designed to involve a larger sample size, and ASA in low dose will be given singly in addition to ART to allow ascertainment of findings directly to it. For studies that investigated the effect of ASA on biomarkers of immune and platelet activation, fewer participants were involved. ${ }^{19} 4041$

The design of our protocol is relevant for attaining the study objectives. Our design is similar to previous studies that looked at the effect of ASA in PLHIV in that randomisation is done. ${ }^{404143}$ However, some of these studies had HIV-uninfected as controls, an aspect lacking in our study design. ${ }^{19}$ Including $\mathrm{HIV}$-uninfected individuals in this study would make the design more complex and introduce ethical dilemma of exposing healthy volunteers to ASA for the duration of the study. In addition, PLHIV as controls are more comparable to PLHIV exposed to ASA than HIV-uninfected individuals. HIV-uninfected individuals may be different from the HIV-infected in certain aspects such as lifestyle, sociodemographic and clinical characteristics.

Our trial is of a parallel design. Some studies have differed in design from our study in that cross-over design was used ${ }^{40} 4243$ and that double blinding was not employed. ${ }^{44}$ Cross-over designs are better suited for studies involving two or more interventions. Whereas a randomised cross-over design could be an option for this trial, randomised double-blind placebo controlled studies are the gold standard in intervention-based studies and are thought to generate evidence of highest quality. ${ }^{54}$

Our study involves HIV-infected patients initiating ART, while previously reported studies were conducted in either ARV-naïve ${ }^{42-44}$ or ARV-treated, virologically 
suppressed HIV-infected patients. ${ }^{19} 41$ Our trial involves ARV-naïve individuals initiating ART rather than the virologically suppressed ones so that the effect of ASA especially on viral load can be determined.

The present study is also designed to assess the level of adherence to ARV drugs in the presence of an additional pill and compliance to study drug by pill count method. Measuring drug levels would be the ideal method of assessing adherence and/or compliance. However, the method is very costly and unavailable in routine HIV management practice in most resource-constrained settings. To ensure that adherence and compliance reflect the nearly true adherence and compliance, pill counts are done on follow-up visits. Throughout the study, the participants are reminded by a phone call and a text message of their upcoming study visit a day before clinic visit, and also they are reminded to carry their drug boxes and bottles with them on the visit day. The participants are also educated on the importance of complying with the study procedure, including adherently taking their study drugs and faithfully returning their study drug boxes and bottles for pill count.

If data from this trial show that ASA is beneficial and does not affect adherence to ART, we will recommend for larger trials and subsequent addition of ASA to the recommended first-line ART. On the other hand, if ASA is shown to have no effect or to be hazardous, a recommendation to avoid ASA in HIV-infected individuals on ART will be made.

\section{Author affiliations}

${ }^{1}$ Department of Clinical Pharmacology, Muhimbili University of Health and Allied Sciences, Dar es Salaam, Tanzania

${ }^{2}$ Department of Internal Medicine, Muhimbili University of Health and Allied Sciences, Dar es Salaam, Tanzania

${ }^{3}$ Department of Community Health, Muhimbili University of Health and Allied Sciences, Dar es Salaam, Dar es Salaam, Tanzania

${ }^{4}$ Department of Epidemiology and Biostatistics, Muhimbili University of Health and Allied Sciences, Dar es Salaam, Tanzania

${ }^{5}$ Department of Microbiology and Immunology, Muhimbili University of Health and Allied Sciences, Dar es Salaam, Tanzania

Acknowledgements The authors thank Miss Ellen Hertzmark for assistance with sample size calculation and randomization. Mr. Mohamed B. Khery for study data management and Mr. Allen J. Mulaki for labeling of the study drug boxes and keeping the blinding.

Collaborators Prof. Takamasa Ueno and Dr. Godfrey Barabona (Joint Research Center for Human Retrovirus Infection, Kumamoto University, Kumamoto, Japan).

Contributors TM, EL, FM, SM and GS developed the study concept and design; TM drafted the initial manuscript; EL, GS, FM, SM, PS, BS and CM reviewed the manuscript. All authors read and approved the final manuscript.

Funding The trial is partially supported by the Fogarty International Center of the National Institutes of Health (NIH) under award numbers R25 TW011227 and 5D43 TW009775-03. The content is solely the responsibility of the authors and does not necessarily represent the official views of the NIH. Cost for analysis of samples for biomarkers of immune and platelet activation and for baseline HIV viral load is covered by our collaborator (Joint Research Center for Human Retrovirus Infection, Kumamoto University, Kumamoto, Japan). The trial is sponsored by MUHAS under the Directorate of Research and Publications (Director of research and publications, Dar es Salaam, Tanzania,E mail:drp@muhas.ac.tz) as the sponsor of the trial with an overall responsibility of ensuring quality and compliance to protocol.

Competing interests None declared.
Patient consent for publication Not applicable.

Provenance and peer review Not commissioned; externally peer reviewed.

Supplemental material This content has been supplied by the author(s). It has not been vetted by BMJ Publishing Group Limited (BMJ) and may not have been peer-reviewed. Any opinions or recommendations discussed are solely those of the author(s) and are not endorsed by BMJ. BMJ disclaims all liability and responsibility arising from any reliance placed on the content. Where the content includes any translated material, BMJ does not warrant the accuracy and reliability of the translations (including but not limited to local regulations, clinical guidelines, terminology, drug names and drug dosages), and is not responsible for any error and/or omissions arising from translation and adaptation or otherwise.

Open access This is an open access article distributed in accordance with the Creative Commons Attribution Non Commercial (CC BY-NC 4.0) license, which permits others to distribute, remix, adapt, build upon this work non-commercially, and license their derivative works on different terms, provided the original work is properly cited, appropriate credit is given, any changes made indicated, and the use is non-commercial. See: http://creativecommons.org/licenses/by-nc/4.0/.

\section{ORCID iDs}

Tosi Mwakyandile http://orcid.org/0000-0002-5073-1923

Bruno Sunguya http://orcid.org/0000-0003-3625-0725

\section{REFERENCES}

1 Joint United Nations Program on HIV/AIDS(UNAIDS). UNAIDS data 2017, 2017.

2 Baker RK, Moorman AC. Mortality in the highly active antiretroviral therapy era; changing causes of death and diseases in the HIV outpatient study. J Acquir Immune Defic Syndr 2006:4327-34.

3 Antiretroviral Therapy Cohort Collaboration. Life expectancy of individuals on combination antiretroviral therapy in high-income countries: a collaborative analysis of 14 cohort studies. Lancet 2008;372:293-9.

4 Rodger AJ, Lodwick R, Schechter M, et al. Mortality in well controlled HIV in the continuous antiretroviral therapy arms of the smart and ESPRIT trials compared with the general population. AIDS 2013:27:973-9

5 Marin B, Thiébaut R, Bucher HC, et al. Non-AIDS-defining deaths and immunodeficiency in the era of combination antiretroviral therapy. AIDS 2009;23:1743-53.

6 , Smith C, Sabin CA, et al, Data Collection on Adverse Events of Anti-HIV drugs (D:A:D) Study Group. Factors associated with specific causes of death amongst HIV-positive individuals in the D:A:D Study. AIDS 2010;24:1537-48.

7 Sackoff JE, Hanna DB, Pfeiffer MR, et al. Causes of death among persons with AIDS in the era of highly active antiretroviral therapy: new York City. Ann Intern Med 2006:145:397-406.

8 Antiretroviral Therapy Cohort Collaboration. Causes of death in HIV-1-infected patients treated with antiretroviral therapy, 19962006: collaborative analysis of 13 HIV cohort studies. Clin Infect Dis 2010;50:1387-96.

9 Islam FM, Wu J, Jansson J, et al. Relative risk of cardiovascular disease among people living with HIV: a systematic review and metaanalysis. HIV Med 2012;13:453-68.

10 Bavinger C, Bendavid E, Niehaus K, et al. Risk of cardiovascular disease from antiretroviral therapy for HIV: a systematic review. PLOS One 2013;8:e59551.

11 Hemkens LG, Bucher HC. Novel therapeutic concepts HIV infection and cardiovascular disease. Eur Heart J 2014;35:1373-81.

12 Lichtenstein KA, Armon C, Buchacz K, et al. Low CD4+ T cell count is a risk factor for cardiovascular disease events in the HIV outpatient study. Clin Infect Dis 2010;51:435-47.

13 Lang S, Mary-Krause M, Simon A, et al. HIV replication and immune status are independent predictors of the risk of myocardial infarction in HIV-infected individuals. Clin Infect Dis 2012;55:600-7.

14 McKibben RA, Margolick JB, Grinspoon S, et al. Elevated levels of monocyte activation markers are associated with subclinical atherosclerosis in men with and those without HIV infection. $J$ Infect Dis 2015;211:1219-28.

15 Burdo TH, Lo J, Abbara S, et al. Soluble CD163, a novel marker of activated macrophages, is elevated and associated with noncalcified coronary plaque in HIV-infected patients. $J$ Infect Dis 2011;204:1227-36.

16 Subramanian S, Tawakol A, Burdo TH, et al. Arterial inflammation in patients with HIV. JAMA 2012;308:379-86. 
17 Kelesidis T, Kendall MA, Yang OO, et al. Biomarkers of microbial translocation and macrophage activation: association with progression of subclinical atherosclerosis in HIV-1 infection. $J$ Infect Dis 2012;206:1558-67.

18 Longenecker CT, Funderburg NT, Jiang Y, et al. Markers of inflammation and CD8 T-cell activation, but not monocyte activation, are associated with subclinical carotid artery disease in HIV-infected individuals. HIV Med 2013;14:385-90.

19 O'Brien M, Montenont E, Hu L, et al. Aspirin attenuates platelet activation and immune activation in HIV-1-infected subjects on antiretroviral therapy: a pilot study. J Acquir Immune Defic Syndr 2013;63:280-8.

20 Shipkova M, Wieland E. Surface markers of lymphocyte activation and markers of cell proliferation. Clin Chim Acta 2012;413:1338-49.

21 Arneth BM. Activation of CD4 and CD8 T cell receptors and regulatory T cells in response to human proteins. PeerJ 2018;6:e4462.

22 Poesen R, Ramezani A, Claes K, et al. Associations of soluble CD14 and endotoxin with mortality, cardiovascular disease, and progression of kidney disease among patients with CKD. Clin J Am Soc Nephrol 2015;10:1525-33.

23 Reiner AP, Lange EM, Jenny NS, et al. Soluble CD14: genomewide association analysis and relationship to cardiovascular risk and mortality in older adults. Arterioscler Thromb Vasc Biol 2013;33:1-18.

24 Longenecker CT, Jiang Y, Orringer CE, et al. Soluble CD14 is independently associated with coronary calcification and extent of subclinical vascular disease in treated HIV infection. AIDS 2014:28:969-77.

25 Blann AD, Nadar SK, Lip GYH, . The adhesion molecule P-selectin and cardiovascular disease. Eur Heart J 2003;24:2166-79.

26 Scialla JJ, Plantinga LC, Kao WHL, et al. Soluble P-selectin levels are associated with cardiovascular mortality and sudden cardiac death in male dialysis patients. Am J Nephrol 2011;33:224-30.

27 Ridker PM, Buring JE, Rifai N. Soluble P-selectin and the risk of future cardiovascular events. Circulation 2001;103:491-5.

28 Hunt PW, Brenchley J, Sinclair E, et al. Relationship between T cell activation and CD4+ T cell count in HIV-seropositive individuals with undetectable plasma HIV RNA levels in the absence of therapy. $J$ Infect Dis 2008;197:126-33.

29 Hunt PW, Martin JN, Sinclair E, et al. T cell activation is associated with lower $\mathrm{CD} 4^{+} \mathrm{T}$ cell gains in human immunodeficiency virusinfected patients with sustained viral suppression during antiretroviral therapy. J Infect Dis 2003;187:1534-43 https://academic.oup.com/ jid/article-lookup/doi/

30 Nkambule BB, Mxinwa V, Mkandla Z, et al. Platelet activation in adult HIV-infected patients on antiretroviral therapy: a systematic review and meta-analysis. BMC Med 2020;18:357.

31 Hattab S, Guiguet M, Carcelain G, et al. Soluble biomarkers of immune activation and inflammation in HIV infection: impact of 2 years of effective first-line combination antiretroviral therapy. HIV Med 2015;16:553-62.

32 Malherbe G, Steel HC, Cassol S. Circulating biomarkers of immune activation distinguish viral suppression from nonsuppression in HAART-treated patients with advanced HIV-1 subtype C infection. Mediators Inflamm 2014:2014:1-7.

33 Wada NI, Jacobson LP, Margolick JB, et al. The effect of HAARTinduced HIV suppression on circulating markers of inflammation and immune activation. AIDS 2015;29:463-71.

34 O'Halloran JA, Dunne E, Gurwith M, et al. The effect of initiation of antiretroviral therapy on monocyte, endothelial and platelet function in HIV-1 infection. HIV Med 2015;16:608-19.

35 Wolf $\mathrm{K}$, Tsakiris DA, Weber R, et al. Antiretroviral therapy reduces markers of endothelial and coagulation activation in patients infected with human immunodeficiency virus type 1. $J$ Infect Dis 2002;185:456-62.

36 Mesquita EC, Hottz ED, Amancio RT, et al. Persistent platelet activation and apoptosis in virologically suppressed HIV-infected individuals. Sci Rep 2018;8:1-10.

37 Falcinelli E, Francisci D, Belfiori B, et al. In vivo platelet activation and platelet hyperreactivity in abacavir-treated HIV-infected patients. Thromb Haemost 2013;110:349-57.

38 Baum PD, Sullam PM, Stoddart CA, et al. Abacavir increases platelet reactivity via competitive inhibition of soluble guanylyl cyclase. AIDS 2011;25:2243-8.

39 Gresele P, Falcinelli E, Momi S, et al. Highly active antiretroviral therapy-related mechanisms of endothelial and platelet function alterations. Rev Cardiovasc Med 2014;15 Suppl 1:S9-20.

40 Falcinelli E, Francisci D, Schiaroli E. Effect of aspirin treatment on abacavir-associated platelet hyperreactivity in HIV-infected patients. Int J Cardiol 2018. doi:10.1016/j.ijcard.2018.04.052

41 O'Brien MP, Hunt PW, Kitch DW, et al. A randomized placebo controlled trial of aspirin effects on immune activation in chronically human immunodeficiency virus-infected adults on virologically suppressive antiretroviral therapy. Open Forum Infect Dis 2017;4:1-10.

42 Stanczuk GA, Thomsen M, Soerensen AM, et al. Acetyl salicylic acid (aspirin), micronutrients and chloroquine in the management of the acquired immunodeficiency syndrome (AIDS). Cent Afr J Med 2002;48:42-9.

43 Sibanda EN, Stanzuk GA, Thomsen M. Acetyl salicylic acid (ASPIRIN) increases the CD4+ Tlymphocytes and suppresses TNF-a in HIV-I infected patients: results of a 12 month, three-arm, placebocontrolled pilot study. AIDS vaccines Relat Top 2004;1:179-90.

44 Durosinmi MA, Armistead H, Akinola NO, et al. Selenium and aspirin in people living with HIV and AIDS in Nigeria. Niger Postgrad Med $J$ 2008;15:215-8.

45 Cantudo-Cuenca MR, Jiménez-Galán R, Almeida-Gonzalez CV, et al. Concurrent use of comedications reduces adherence to antiretroviral therapy among HIV-infected patients. J Manag Care Spec Pharm 2014;20:844-50.

46 Horberg MA, Silverberg MJ, Hurley LB, et al. Effects of depression and selective serotonin reuptake inhibitor use on adherence to highly active antiretroviral therapy and on clinical outcomes in HIV-infected patients. J Acquir Immune Defic Syndr 2008;47:384-90.

47 Palepu A, Tyndall MW, Joy R, et al. Antiretroviral adherence and HIV treatment outcomes among HIV/HCV co-infected injection drug users: the role of methadone maintenance therapy. Drug Alcohol Depend 2006;84:188-94.

48 United Republic of Tanzania. World population policies 2019, 2020.

49 Drake BJ, Sarah D, Tina C, et al. CD4 count recovery after antiretroviral therapy initiation in patients infected with the human immunodeficiency virus. Am J Med Sci [Internet] 2016.

50 Sangeda RZ, Mosha F, Prosperi M, et al. Pharmacy refil adherence outperforms self-reported methods in predicting HIV therapy outcome in resource-limited settings. BMC Public Health 2014;14:1-11.

51 Soomro H, Aleem S, Hussain M, et al. Frequency and predictors of non-compliance to aspirin therapy in post myocardial infarction patients. Glob J Health Sci 2017;9:217-23.

52 Walmsley SL, Antela A, Clumeck N, et al. Dolutegravir plus abacavirlamivudine for the treatment of HIV-1 infection. N Engl J Med 2013;369:1807-18

53 Huang ES, Strate LL, Ho WW, et al. Long-term use of aspirin and the risk of gastrointestinal bleeding. Am J Med 2011:124:426-33

54 Misra S. Randomized double blind placebo control studies, the "gold standard" in intervention based studies. Indian J Sex Transm Dis 2012;33:131-4. 